\title{
Nota
}

\section{EFICIÊNCIA SIMBIÓTICA DE ISOLADOS DE RIZÓBIO NODULADORES DE FEIJÃO-FAVA (Phaseolus lunatus L.) ${ }^{(1)}$}

\author{
Jadson Emanuel Lopes Antunes ${ }^{(2)}$, Regina Lúcia Ferreira Gomes ${ }^{(3)}$, Ângela \\ Celis de Almeida Lopes ${ }^{(4)}$, Ademir Sérgio Ferreira de Araújo ${ }^{(5)}$, Maria do Carmo \\ Catanho Pereira de Lyra $^{(6)}$ \& Márcia do Vale Barreto Figueiredo ${ }^{(7)}$
}

\begin{abstract}
RESUMO
Em leguminosas tropicais, a cuidadosa seleção de estirpes de rizóbio, entre outros fatores, é fundamental para a eficiência da fixação biológica de $\mathrm{N}_{2}$ (FBN). Essa seleção deve ser feita para as leguminosas de interesse social e econômico, entre elas o feijão-fava (Phaseolus lunatus L.). O objetivo deste trabalho foi avaliar a eficiência simbiótica de rizóbios nativos de duas regiões do Piauí produtoras de feijão-fava. Foram avaliados 17 isolados e duas estirpes de referência (CIAT 899 e NGR 234), em casa de vegetação, utilizando-se vasos de Leonard autoclavados, no delineamento experimental inteiramente ao acaso, com três repetições. O genótipo de feijão-fava utilizado foi o UFPI-468. A inoculação foi feita por ocasião do plantio. A coleta foi realizada aos 34 dias após o plantio, sendo avaliadas as seguintes características: matéria seca da parte aérea (MSPA), da raiz (MSR) e dos nódulos (MSN); relação MSPA/MSR; N acumulado (Nac) na MSPA e a eficiência da fixação de $\mathrm{N}_{2}$. Foi observada diferença significativa entre os isolados em todas as características, exceto em MSR. Em geral, os isolados ISO-18, ISO-23, ISO-24, ISO25, ISO-30, ISO-32, ISO-35, ISO-36, ISO-43 e ISO-45 apresentaram os melhores índices de MSPA, MSR, MSPA/MSR, Nac e eficiência da fixação de $\mathrm{N}_{2}$, em relação aos
\end{abstract}

(1) Parte da Dissertação de Mestrado do primeiro autor apresentada ao Programa de Pós-Graduação em Agronomia (PPGA), Centro de Ciências Agrárias (CCA), Universidade Federal do Piauí - UFPI. Recebido para publicação em abril de 2010 e aprovado em fevereiro de 2011.

(2) Mestrando do PPGA da Universidade Federal do Piauí - UFPI. Campus Ministro Petrônio Portela, s/n, Bairro Ininga, CEP 64049550 Teresina (PI). E-mail: jadsonemanuel@gmail.com

(3) Departamento de Fitotecnia, CCA, UFPI. E-mail: rlfgomes@ufpi.edu.br

(4) Departamento de Biologia, Centro de Ciências da Natureza (CCN), UFPI. Bolsista de Produtividade de Pesquisa do CNPq. Email: acalopes@ufpi.edu.br

(5) Departamento de Engenharia Agrícola e Solos. CCA, UFPI. Bolsista de Produtividade de Pesquisa do CNPq. E-mail: asfaruaj@yahoo.com.br

${ }^{(6)}$ Laboratório de Genômica, Instituto Agronômico de Pernambuco - IPA. Av. San Martin 1331, Bonji, CEP 50761-000 Recife (PE). E-mail: maria.catanho@ipa.br

(7) Laboratório de Biologia do Solo, Instituto Agronômico de Pernambuco - IPA. Bolsista de Produtividade de Pesquisa do CNPq. E-mail: marcia.figueiredo@ipa.br 
isolados ISO-2, ISO-9, ISO-16, ISO-40 e testemunha absoluta. As características avaliadas foram suficientes para discriminar e selecionar isolados eficientes na nodulação em feijão-fava, contribuindo para a efetividade da FBN. Os melhores isolados apresentaram bom desempenho no fornecimento de $\mathrm{N}$ às plantas, podendo ser recomendados para testes de eficiência agronômica.

Termos de indexação: fixação biológica do nitrogênio, nodulação, simbiose, Bradyrhizobium sp.

\title{
SUMMARY: SYMBIOTIC EFFICIENCY OF RHIZOBIA ISOLATED FROM NODULES OF LIMA BEAN (Phaseolus lunatus L.)
}

\begin{abstract}
In tropical legumes, a careful selection of rhizobia strains, among other factors, is essential for the efficiency of biological nitrogen fixation (BNF). This selection should be made for leguminous crops of social and economic interest, such as lima bean (Phaseolus lunatus L.). The aim of this study was to evaluate the symbiotic effectiveness of rhizobia from two regions of Piaui producing lima bean. Seventeen isolates and two reference strains CIAT 899 and NGR 234 were evaluated on the fava bean genotype "UFPI-468". The study was conducted in a greenhouse in autoclaved Leonard jars, in a randomized block design with three replications. Inoculation was done at planting. Samples were collected 34 days after planting to assess the following variables: shoot dry mass (SDM), root (MRS) and nodules (MNS), ratio SDM/MRS, accumulated nitrogen (Nac) in SDM by the Kjeldahl method, and $\mathrm{N}_{2}$-fixation efficiency. Significant interactions were observed among all variables except for MSR. In general, the values for DMAP, MRS , DMAP/MRS, Nac and $\mathrm{N}_{2}$-fixation efficiency of the isolates ISO-18, ISO-23, ISO-24, ISO-25, ISO-30, ISO-32, ISO-35, ISO-36, ISO-43, and ISO-45 were higher than of ISO-2, ISO-9, ISO 16, and ISO-40 and the absolute control. The variables used in this study were sufficient to discriminate and select nodulation-efficient strains for lima bean, increasing the effectiveness of BNF. Most isolates performed well in terms of $N$ supply to plants and can be recommended for tests of agronomic efficiency.
\end{abstract}

Index terms: biological nitrogen fixation, nodulation, symbiosis, Bradyrhizobium sp.

\section{INTRODUÇÃO}

Dos sistemas biológicos envolvendo plantas e microrganismos, a simbiose rizóbio-leguminosa é o de maior expressão econômica (Franco et al., 2002), pois traz benefícios para a sustentabilidade agrícola (Xavier et al., 2006), por meio do processo de fixação biológica do $\mathrm{N}_{2}(\mathrm{FBN})$.

Um grande número de espécies de leguminosas é capaz de se associar, simbioticamente, com bactérias fixadoras de $\mathrm{N}_{2}$, denominadas, coletivamente, de rizóbio. Entretanto, a eficiência da fixação é variável, sendo influenciada, dentre outros fatores, pela especificidade hospedeira. Na seleção de estirpes de rizóbios eficientes, várias etapas são necessárias, como a verificação da capacidade de nodulação da espécie, o isolamento das bactérias, a purificação das colônias e a avaliação da eficiência em vasos com substratos esterilizados e solo não esterilizado (Faria \& Franco, 2002).

A seleção de estirpes de rizóbio para diferentes leguminosas tem sido objeto de vários estudos (Neves et al., 1998a,b; Hungria et al., 2001; Hara \& Oliveira, 2005; Zaman-Hallah et al., 2007). A seleção de estirpes eficientes para a FBN em feijão-fava deve considerar, além da eficiência da estirpe, características como habilidade de competição e sobrevivência no solo, capacidade de formação de nódulos, estabilidade genética e fixação na espécie alvo (Lima et al., 2005; Moreira \& Siqueira, 2006; Zilli et al., 2006).

O número, a biomassa e o tamanho dos nódulos são indicadores usuais de nodulação (Ferreira \& Castro, 1995) e constituem critérios utilizados para avaliação da simbiose rizóbio-leguminosas, fazendo parte, inclusive, do protocolo para avaliação da eficiência agronômica de estirpes no Brasil pela Rede de Laboratórios para Recomendação, Padronização e Difusão de Tecnologia de Inoculantes Microbianos de Interesse Agrícola (RELARE) (Xavier et al., 2006). Além disso, Souza et al. (2008) definiram um conjunto mínimo de dados para avaliação da FBN, como a massa dos nódulos e da parte aérea e o total de $\mathrm{N}$ acumulado pela planta.

O feijão-fava (Phaseolus lunatus L.) é uma das espécies do gênero Phaseolus com maior importância 
econômica para a região Nordeste do Brasil e capaz de realizar a FBN (Santos et al., 2008). Contudo, a FBN em feijão-fava tem sido pouco estudada, e as pesquisas com gênero Phaseolus têm enfocado o $P$. vulgaris, com estirpes de rizóbio recomendadas para uso comercial. No Brasil, não há isolados ou estirpes selecionadas para a inoculação do $P$. lunatus, o que reforça a necessidade de isolar, caracterizar e selecionar bactérias nativas capazes de nodular essa espécie (Santos, 2008). Nesse contexto, o objetivo deste trabalho foi avaliar a eficiência simbiótica de rizóbios nativos de áreas produtoras de feijão-fava do Estado do Piauí.

\section{MATERIAL E MÉTODOS}

O trabalho foi conduzido em casa de vegetação do Instituto Agronômico de Pernambuco (IPA), de julho a agosto de 2009 , sendo utilizados 17 isolados e duas estirpes de referência: Rhizobium tropici CIAT 899 (SEMIA 4077) e Rhizobium sp. NGR 234 (SEMIA 6357). A CIAT 899 foi utilizada por ser atualmente recomendada pelo Ministério da Agricultura, Pecuária e Abastecimento para composição de inoculante comercial para a cultura do feijão-comum (Phaseolus vulgaris) (Hungria et al., 1997; Chueire et al., 2003), que pertence ao mesmo gênero do feijão-fava (Phaseolus lunatus). A NGR 234 foi utilizada por ser descrita como uma estirpe que nodula mais de 112 gêneros de leguminosas (Trinick, 1980; Giller \& Wilson, 1993).
Essas estirpes foram cedidas pelo Laboratório de Genoma do IPA.

As classificações morfológicas e fisiológicas dos isolados utilizados neste estudo foram realizadas por Santos (2008) (Quadro 1).

Os isolados de crescimento rápido e as estirpes de referência foram crescidos em duplicatas, em erlenmeyers de $125 \mathrm{~mL}$, contendo $50 \mathrm{~mL}$ de meio líquido (YEM - manitol e extrato de levedura (Vincent, 1970) a $250 \mathrm{rpm}$ e $28^{\circ} \mathrm{C}$, por $72 \mathrm{~h}$. Os isolados de crescimento intermediário permaneceram nas mesmas condições por $120 \mathrm{~h}$. A concentração de bactérias utilizada foi de aproximadamente $10^{9}$ $\mathrm{UFC} \mathrm{mL}^{-1}$, na fase de crescimento exponencial.

O delineamento experimental foi o inteiramente casualizado, com 20 tratamentos (17 isolados, duas estirpes de referência e uma testemunha absoluta (TA) sem $\mathrm{N}$ e sem inoculação) e três repetições. Na instalação do experimento, foram utilizadas sementes de feijão-fava genótipo “UFPI-468”, de tegumento begeclaro, oriunda do Banco Ativo de Germoplasma de Feijão-Fava (BAG de Feijão-Fava) da UFPI. As sementes foram desinfestadas com álcool a $70 \%$ por $30 \mathrm{~s}$, depois com hipoclorito de sódio a $2 \%$ por $60 \mathrm{~s}$, seguido de sete lavagens sucessivas com $\mathrm{H}_{2} \mathrm{O}$ destilada autoclavada (Vincent, 1970). Para o cultivo das plantas foram utilizados vasos de Leonard contendo areia + vermiculita (1.300 g da mistura) ( $\mathrm{pH} 6,8)$ na proporção de $2: 1$, autoclavado por uma hora, em temperatura de $120^{\circ} \mathrm{C}$, a $101 \mathrm{kPa}$.

Quadro 1. Características morfológicas e fisiológicas dos isolados de rizóbios, oriundos dos solos de Nova Esperança e Santa Rita, município de Água Branca, PI noduladores de feijão-fava (Phaseolus lunatus L.)

\begin{tabular}{|c|c|c|c|c|c|c|c|c|c|c|}
\hline Isolados & Origem $^{(1)}$ & Cultivar $^{(2)}$ & $\mathrm{FC}^{(3)}$ & $\mathrm{EL}^{(4)}$ & $\mathrm{CC}^{(5)}$ & $\mathrm{TC}^{(6)}$ & $\mathbf{F A A}^{(7)}$ & $\mathrm{FM}^{(8)}$ & $\mathrm{VM}^{(9)}$ & $\mathrm{EM}^{(10)}$ \\
\hline ISO - 2 & $\mathrm{NE}$ & $\mathrm{BM}$ & $\mathrm{R}$ & $\mathrm{C}$ & $\mathrm{T}$ & $\mathrm{R}$ & $\mathrm{Ac}$ & $\mathrm{P}$ & $\mathrm{P}$ & $\mathrm{P}$ \\
\hline ISO - 9 & $\mathrm{NE}$ & $\mathrm{BM}$ & $\mathrm{R}$ & $\mathrm{C}$ & $\mathrm{BL}$ & $\mathrm{R}$ & Ac & $\mathrm{P}$ & $\mathrm{P}$ & $\mathrm{P}$ \\
\hline ISO - 16 & SR & $\mathrm{BM}$ & $\mathrm{R}$ & $\mathrm{C}$ & $\mathrm{T}$ & $\mathrm{I}$ & $\mathrm{Ac}$ & $\mathrm{P}$ & MU & $\mathrm{P}$ \\
\hline ISO - 18 & SR & FM & $\mathrm{R}$ & $\mathrm{C}$ & $\mathrm{T}$ & I & $\mathrm{Al}$ & $\mathrm{P}$ & MU & $\mathrm{A}$ \\
\hline ISO - 21 & SR & FM & $\mathrm{R}$ & $\mathrm{C}$ & $\mathrm{BL}$ & $\mathrm{R}$ & Ac & $\mathrm{P}$ & MU & $\mathrm{P}$ \\
\hline ISO - 23 & SR & FM & $\mathrm{R}$ & $\mathrm{C}$ & $\mathrm{BL}$ & $\mathrm{I}$ & $\mathrm{Al}$ & $\mathrm{P}$ & MU & A \\
\hline ISO - 24 & SR & FM & $\mathrm{R}$ & $\mathrm{C}$ & $\mathrm{BL}$ & I & $\mathrm{Al}$ & $\mathrm{P}$ & $\mathrm{M}$ & $\mathrm{A}$ \\
\hline ISO - 25 & SR & FM & $\mathrm{R}$ & $\mathrm{C}$ & $\mathrm{BL}$ & I & $\mathrm{Al}$ & $\mathrm{P}$ & MU & $\mathrm{A}$ \\
\hline ISO - 27 & SR & FM & $\mathrm{E}$ & $\mathrm{C}$ & $\mathrm{BL}$ & I & Ac & $\mathrm{P}$ & MU & $\mathrm{A}$ \\
\hline ISO - 30 & SR & FM & $\mathrm{R}$ & $\mathrm{A}$ & $\mathrm{BL}$ & $\mathrm{R}$ & $\mathrm{Al}$ & $\mathrm{P}$ & M & $\mathrm{A}$ \\
\hline ISO - 32 & $\mathrm{NE}$ & FM & $\mathrm{R}$ & $\mathrm{C}$ & $\mathrm{BL}$ & I & $\mathrm{Al}$ & $\mathrm{P}$ & MU & A \\
\hline ISO - 35 & $\mathrm{NE}$ & FM & $\mathrm{R}$ & $\mathrm{C}$ & $\mathrm{BL}$ & I & $\mathrm{Al}$ & $\mathrm{P}$ & MU & A \\
\hline ISO - 36 & $\mathrm{NE}$ & FM & $\mathrm{R}$ & $\mathrm{C}$ & $\mathrm{BL}$ & $\mathrm{R}$ & $\mathrm{Ac}$ & $\mathrm{P}$ & M & $\mathrm{A}$ \\
\hline ISO - 40 & $\mathrm{NE}$ & FM & $\mathrm{R}$ & A & $\mathrm{BL}$ & $\mathrm{R}$ & Ac & $\mathrm{P}$ & MU & A \\
\hline ISO - 43 & $\mathrm{NE}$ & FM & $\mathrm{R}$ & $\mathrm{C}$ & $\mathrm{BL}$ & $\mathrm{R}$ & $\mathrm{Al}$ & $\mathrm{P}$ & $\mathrm{P}$ & $\mathrm{A}$ \\
\hline ISO - 45 & $\mathrm{NE}$ & FM & $\mathrm{R}$ & $\mathrm{A}$ & $\mathrm{BL}$ & I & Ac & $\mathrm{P}$ & MU & $\mathrm{A}$ \\
\hline ISO - 50 & $\mathrm{NE}$ & FM & $\mathrm{R}$ & $\mathrm{C}$ & $\mathrm{BL}$ & I & Ac & $\mathrm{P}$ & UM & $\mathrm{A}$ \\
\hline
\end{tabular}

(1) Localidade(NE: Nova Esperança-PI, SR: Santa Rita-PI). ${ }^{(2)}$ Cultivar (BM: Boca de Moça (UFPI-468); FM: Fava Miúda (UFPI491). ${ }^{(3)}$ Forma da colônia(R: redondas,E: elipsoide). ${ }^{(4)}$ Elevação (C: convexa, A: achatada). ${ }^{(5)}$ Cor (BL: Branca/leitosa,T: transparente). ${ }^{(6)}$ Tempo de crescimento(R: rápido, I: intermediário). ${ }^{(7)}$ Formação de ácido e álcalis (Ac: ácida, Al: alcalina). ${ }^{(8)}$ formação de muco (A: ausente, P: presente). ${ }^{(9)}$ Volume do muco (S: seca, P: pouco, M: médio e MU: muito). ${ }^{(10)}$ Elasticidade do muco (P: presença de fio, A: ausência de fio). 
O plantio foi feito adicionando-se, nos vasos Leonard com o substrato, sementes inoculadas com $1 \mathrm{~mL}$ do meio de cultura. Seis dias após o plantio, foi efetuado o desbaste, deixando-se duas plantas por vaso. Foi utilizada a solução nutritiva de Hoagland \& Arnon (1950), modificada conforme Silveira et al. (1998). Aos 12 dias após a semeadura, foi verificada a presença de larvas de trips, bem como de adultos de mosca branca causando danos às plantas. Como medida de controle, as plantas foram pulverizadas com óleo de Neem indiano a $1 \%$.

A coleta foi realizada aos 34 dias após o plantio, sendo avaliados as seguintes características: matéria seca da parte aérea (MSPA), da raiz (MSR) e dos nódulos (MSN); relação MSPA/MSR; N acumulado (Nac) na MSPA, pelo método de Kjeldahl, segundo Bremner (1965); e a eficiência da fixação de $\mathrm{N}_{2}$ (Faria \& Franco, 2002) Na coleta, a parte aérea das plantas foi acondicionada em sacos de papel, que foram deixados em estufa de circulação forçada a $65^{\circ} \mathrm{C}$, durante $72 \mathrm{~h}$, para se proceder à avaliação da matéria seca da parte aérea (MSPA). Os nódulos foram secos nas mesmas condições anteriores e pesados, e as raízes, após coleta dos nódulos e limpeza de todos os resquícios de substrato, foram secas também em estufa a $65^{\circ} \mathrm{C}$.

Os dados foram submetidos à análise de variância pelo teste $\mathrm{F}(\mathrm{p}<0,05)$, sendo as médias comparadas pelo teste de Tukey $(\mathrm{p}<0,05)$, utilizando-se o programa estatístico SAEG, versão 8.0 (SAEG, 2000).

\section{RESULTADOS E DISCUSSÃO}

Os isolados ISO-18, ISO-21, ISO-23, ISO-24, ISO25, ISO-30, ISO-32, ISO-35, ISO-36, ISO-43, ISO-45 e ISO-50 apresentaram valores de matéria seca da parte aérea (MSPA) semelhantes aos das estirpes de referência CIAT 899 e NGR 234 (Quadro 2). Os isolados ISO-18, ISO-30 e ISO-32 destacaram-se por apresentar maiores valores de MSPA do que os isolados ISO-2, ISO-9, ISO-16, ISO-40 e a testemunha absoluta (TA). Em comparação à TA, os isolados ISO-18, ISO30 e ISO-32 apresentaram valores aproximadamente duas vezes superiores.

A avaliação da MSPA representa um bom indicativo do estado nutricional das plantas, sendo importante para proporcionar à cultura grande potencial de produção (Xavier et al., 2006; Souza et al., 2008). Essa característica vem sendo utilizada na seleção de estirpes para composição de inoculantes (Zilli et al., 2006; Souza et al., 2008). Os resultados encontrados para a MSPA indicam que a maioria dos isolados proporcionou crescimento adequado da

Quadro 2. Valores médios da matéria seca da parte aérea (MSPA), da raiz (MRS), do nódulo (MNS), da relação (MSPA/MRS), do nitrogênio acumulado na MSPA (Nac) e da eficiência da fixação do $\mathrm{N}_{2}$ dos diferentes isolados de rizóbios inoculados em feijão-fava (Phaseolus lunatus L.) genótipo UFPI-468

\begin{tabular}{|c|c|c|c|c|c|c|}
\hline Tratamento & MSPA & MRS & MNS & MSPA/MRS & Nac & $\begin{array}{l}\text { Eficiência da } \\
\text { fixação de } \mathbf{N}_{2}{ }^{(1)}\end{array}$ \\
\hline & & g/vaso & - & $\mathrm{g} \mathrm{g}^{-1}$ & $\mathrm{mg} /$ vaso de $\mathrm{N}$ & $\%$ \\
\hline ISO - 2 & $2,58 \mathrm{cde}$ & $0,76 \mathrm{a}$ & 0,13 cde & $3,20 \mathrm{def}$ & $28,94 \mathrm{de}$ & 89 \\
\hline ISO - 9 & $2,30 \mathrm{de}$ & $0,73 \mathrm{a}$ & 0,21 bcde & 3,41 ef & 41,11 cde & 79 \\
\hline ISO - 16 & $2,00 \mathrm{e}$ & $0,65 \mathrm{a}$ & $0,06 \mathrm{de}$ & $3,06 \mathrm{f}$ & $20,17 \mathrm{e}$ & 69 \\
\hline ISO - 18 & $6,57 \mathrm{ab}$ & $0,75 \mathrm{a}$ & $0,63 \mathrm{abc}$ & $9,06 \mathrm{a}$ & 227,95 a & 226 \\
\hline IS O- 21 & 5,66 abcd & $0,75 \mathrm{a}$ & 0,48 abcde & 7,49 abcde & $162,87 \mathrm{abcd}$ & 195 \\
\hline ISO - 23 & 5,26 abcde & $0,64 \mathrm{a}$ & $0,65 \mathrm{ab}$ & $8,21 \mathrm{ab}$ & $223,86 \mathrm{a}$ & 181 \\
\hline ISO - 24 & 4,98 abcde & $0,67 \mathrm{a}$ & $0,57 \mathrm{abc}$ & 7,49 abcde & $195,14 \mathrm{a}$ & 172 \\
\hline ISO - 25 & $5,91 \mathrm{abc}$ & $0,77 \mathrm{a}$ & $0,65 \mathrm{ab}$ & 7,82 abcd & $205,16 \mathrm{a}$ & 204 \\
\hline ISO - 27 & 3,70 bcde & $0,83 \mathrm{a}$ & 0,51 abcd & 4,52 bcdef & 97,21 abcde & 127 \\
\hline ISO - 30 & $7,29 \mathrm{a}$ & $0,91 \mathrm{a}$ & $0,72 \mathrm{ab}$ & $8,29 \mathrm{ab}$ & $219,04 \mathrm{a}$ & 251 \\
\hline ISO - 32 & $6,61 a b$ & $0,80 \mathrm{a}$ & $0,58 \mathrm{abc}$ & $8,41 \mathrm{ab}$ & $205,63 \mathrm{a}$ & 229 \\
\hline ISO - 35 & $5,91 \mathrm{abc}$ & $0,89 \mathrm{a}$ & $0,54 \mathrm{abcd}$ & 6,82 abcdef & $201,48 \mathrm{a}$ & 204 \\
\hline ISO - 36 & $5,91 \mathrm{abc}$ & $0,76 \mathrm{a}$ & $0,66 \mathrm{ab}$ & $7,92 \mathrm{abc}$ & $177,56 \mathrm{abc}$ & 204 \\
\hline IS O - 40 & 2,81 cde & $0,75 \mathrm{a}$ & 0,13 cde & 3,77 cdef & 44,22 bcde & 97 \\
\hline ISO - 43 & $5,95 \mathrm{abc}$ & $0,77 \mathrm{a}$ & $0,66 \mathrm{ab}$ & 7,75 abcd & $224,24 \mathrm{a}$ & 205 \\
\hline ISO - 45 & $5,97 \mathrm{abc}$ & $0,84 \mathrm{a}$ & $0,77 \mathrm{a}$ & 7,07 abcdef & $186,69 \mathrm{ab}$ & 206 \\
\hline ISO - 50 & 5,25 abcde & $0,78 \mathrm{a}$ & $0,70 \mathrm{ab}$ & 6,84 abcdef & $170,56 \mathrm{abcd}$ & 181 \\
\hline CIAT - 899 & 5,15 abcde & $0,70 \mathrm{a}$ & $0,63 \mathrm{abc}$ & 7,37 abcdef & $193,40 \mathrm{a}$ & 177 \\
\hline NGR 234 & $5,87 \mathrm{abc}$ & $0,78 \mathrm{a}$ & $0,66 \mathrm{ab}$ & 7,36 abcdef & 147,87 abcde & 202 \\
\hline $\mathrm{TA}$ & 2,90 cde & $0,83 \mathrm{a}$ & $0,00 \mathrm{e}$ & 3,47 def & $29,95 \mathrm{de}$ & 100 \\
\hline CV (\%) & 22,83 & 18,63 & 28,45 & 21,8 & 30,34 & - \\
\hline
\end{tabular}

(1) Eficiência da fixação de $\mathrm{N}_{2}$ (MSPA trat/peso da testemunha absoluta x 100) (Faria \& Franco, 2002). Na coluna, médias (três repetições) seguidas pelas mesmas letras não diferem estatisticamente $(p<0,05)$ pelo teste de Tukey. 
cultura. Além disso, os valores de MSPA do feijãofava reforçam a necessidade de estudos com esses isolados em vasos com solo e campo visando a futuras indicações para essa cultura.

Não foram observadas diferenças significativas, entre os tratamentos, para a matéria seca das raízes (MSR) (Quadro 2), sugerindo que os isolados não exerceram influência sobre o crescimento radicular do feijão-fava.

Houve grande variação nos valores de matéria dos nódulos secos (MSN) (Quadro 2), evidenciando que entre os isolados estudados existem grandes diferenças para a capacidade de formar nódulos no feijão-fava. Esses resultados discordam dos obtidos por Nogueira (2005) em feijão-comum, que observou pequena variação para a matéria seca dos nódulos entre rizóbios. Nesse caso, a pequena variação é explicada pelos longos estudos sobre avaliação de estirpes em feijão- comum. Para feijão-fava, os resultados mostram a necessidade de intensificar os estudos com isolados para a cultura.

Os maiores valores para a MSN foram observados nos isolados ISO-18, ISO-21, ISO-23, ISO-24, ISO-25, ISO-27, ISO-30, ISO-32, ISO-35, ISO-36, ISO-43, ISO45 e ISO-50 e nas estirpes de referência (Quadro 2). Os menores valores para a MSN foram observados nos isolados ISO-2, ISO-16 e ISO-40. Na TA não foram encontrados nódulos, indicando que não houve contaminação do experimento.

O número e a massa dos nódulos são indicativos de nodulação (Ferreira \& Castro, 1995; Araújo et al., 2007). Entretanto, a massa dos nódulos é mais útil na avaliação da nodulação, em virtude da melhor correlação com o desempenho simbiótico (Döbereiner, 1966; Bohrer \& Hungria, 1998; Hungria \& Bohrer, 2000). Nesse sentido, os isolados ISO-23, ISO-25, ISO30, ISO-36, ISO-43, ISO-45 e ISO-50 mostraram melhor desempenho simbiótico com o feijão-fava.

O baixo valor de MSN dos tratamentos com os isolados ISO-2, ISO-16 e ISO-40 pode ter ocorrido devido à baixa interação entre os isolados e o genótipo do feijão-fava utilizado. Moreira \& Siqueira (2006) reportam que a formação dos nódulos depende dos genótipos das plantas e das estirpes ou isolados envolvidos nessa interação, além dos fatores ambientais.

As plantas de feijão-fava inoculadas com os isolados ISO-18, ISO-21, ISO-23, ISO-24, ISO-25, ISO-30, ISO32, ISO-35, ISO-36, ISO-43, ISO-45 e ISO-50 mostraram maior relação MSPA/MSR do que os valores observados para os isolados ISO-2, ISO-9, ISO16, ISO-27 e ISO-40 (Quadro 2). Ademais, aqueles isolados apresentaram valores semelhantes aos das estirpes de referência. Os isolados ISO-18, ISO-23, ISO-30 e ISO-32 apresentaram valores de MSPA/MSR aproximadamente 2,5 superiores ao obtido na testemunha.

Os isolados ISO-18, ISO-23, ISO-24, ISO-25, ISO30, ISO-32, ISO-35 e ISO-43 e as estirpes de referência
CIAT 899 e NGR 234 proporcionaram maior acúmulo de $\mathrm{N}$ (Nac) nas plantas (Quadro 2), com valores superiores a $190 \mathrm{mg} / \mathrm{vaso}$ de N. Esse aumento no Nac foi em torno de sete vezes superior ao valor obtido pela testemunha. Por sua vez, os isolados ISO-2, ISO-9, ISO-16 e ISO-40 proporcionaram o menor acúmulo de $\mathrm{N}$ para as plantas, com valores inferiores a $45 \mathrm{mg} /$ vaso de $\mathrm{N}$.

Os resultados observados para o Nac indicam que os isolados ISO-18, ISO-23, ISO-24, ISO-25, ISO-30, ISO-32, ISO-35 e ISO-43 apresentaram satisfatória capacidade de fixação de $\mathrm{N}_{2}$, uma vez que não houve fertilização nitrogenada para a planta. Além disso, como os valores de Nac nos tratamentos com esses isolados foram superiores ao observado na testemunha absoluta, considera-se que a maior proporção de $\mathrm{N}$ encontrada nas plantas foi proveniente da fixação biológica do $\mathrm{N}$ e de pequena parte da reserva da semente (Fernandes et al., 2003). Esses mesmos isolados proporcionaram maior acúmulo de MSPA e MNS, o que indica correlação positiva entre essas variáveis (Quadro 3).

Correlações positivas e significativas entre a massa nodular e a quantidade de $\mathrm{N}_{2}$ fixado biologicamente foram relatadas também por Döbereiner et al. (1966), Santos (1987), Bohrer \& Hungria (1998) e Fernandes \& Fernandes (2000) e indicam a importância da massa dos nódulos na eficiência da fixação de $\mathrm{N}_{2}$ em leguminosas.

Quadro 3. Matriz de correlação entre a matéria seca da parte aérea (MSPA), da raiz (MSR), do nódulo (MSN), da relação (MSPA/MSR), do nitrogênio acumulado na MSPA (Nac) e da eficiência de fixação do nitrogênio, avaliados nos isolados de rizóbios inoculados em feijão-fava (Phaseolus lunatus L.), genótipo UFPI-468

\section{MSR MSN MSPA/MSR NAC $\begin{aligned} & \text { Eficiência da } \\ & \text { fixação de } \mathrm{N}_{2}\end{aligned}$}

\begin{tabular}{|c|c|c|c|c|c|}
\hline MSPA & 0,3544 & $0,8891^{*}$ & 0,9504 & $0,9369^{*}$ & $0,9999^{*}$ \\
\hline MSR & & 0,1944 & 0,0635 & 0,1255 & 0,3550 \\
\hline MSN & & & $0,8875^{*}$ & $0,9133^{*}$ & $0,8886^{*}$ \\
\hline MSPA/MSR & & & & $0,9614^{*}$ & $0,9501^{*}$ \\
\hline NAC & & & & & $0,9369^{*}$ \\
\hline
\end{tabular}

*: significativo a $5 \%$.

\section{CONCLUSÃO}

Os isolados estudados apresentam capacidade e eficiência na nodulação e na fixação de $\mathrm{N}_{2}$ em feijãofava, porém estudos devem ser conduzidos para avaliar a capacidade de fixação de $\mathrm{N}_{2}$, em condições de vasos com solos e de campo, a fim de se comprovar o seu potencial para uso na composição de inoculantes microbianos para indicação na cultura estudada. 


\section{AGRADECIMENTOS}

À CAPES, pela concessão de bolsa de mestrado ao primeiro autor; ao $\mathrm{CNPq}$, pelo auxílio às pesquisas com Phaseolus lunatus; e ao Instituto Agronômico de Pernambuco - IPA.

\section{LITERATURA CITADA}

ARAUJO, F.F.; CARMONA, F.G.; TIRITAN, C.S. \& CRESTE, J.E. Fixação biológica de $\mathrm{N}_{2}$ no feijoeiro submetido a dosagens de inoculante e tratamento químico na semente comparado à adubação nitrogenada. Act. Sci. Agron., 29:535-540, 2007.

BOHRER, T.R.J. \& HUNGRIA, M. Avaliação de cultivares de soja quanto à fixação biológica do nitrogênio. Pesq. Agropec. Bras., 33:937-952, 1998.

BREMNER, J.M. Total nitrogen. In: BLACK, C.A., ed. Methods of soil analysis chemical and microbiological properties. Madison, American Society of Agronomy, 1965. Part 2. p.1149-1178.

CHUEIRE, L.M.O.; BANGEL, E.V.; MOSTASSO, R.J.; PEDROSA, F.O. \& HUNGRIA, M. Classificação taxonômica das estirpes de rizóbio recomendadas para as culturas da soja e do feijoeiro baseada no seqüenciamento do gene 16S rRNA. R. Bras. Ci. Solo, 27:833-840, 2003.

DÖBEREINER, J. Evaluation of nitrogen fixation in legumes by the regression of total plant nitrogen with nodule weight. Nature, 210:850-852, 1966.

FARIA, S.M. \& FRANCO, A.A. Identificação de bactérias eficientes na fixação biológica de nitrogênio para espécies leguminosas arbóreas. Seropédica, Embrapa Agrobiologia, 2002. 16 p. (Documentos, 158). FERNANDES, M.F. \& FERNANDES, R.P.M. Seleção inicial e caracterização parcial de rizóbios de tabuleiros costeiros quando associados ao guandu. R. Bras. Ci. Solo, 24:321-327, 2000.

FERNANDES, M.F.; FERNANDES, R.P.M. \& HUNGRIA, M. Seleção de rizóbios nativos para guandu, caupi e feijãode-porco nos tabuleiros costeiros de Sergipe. Pesq. Agropec. Bras., 38:835-842, 2003.

FERREIRA, E.M. \& CASTRO, I.V. Nodulation and growth of subterranean clover (Trifolium subterraneum L.) in soils previously treated with sewage sludge. Soil Biol. Biochem., 27:177-1183, 1995.

FRANCO, M.C.; CASSINI, S.T.A.; OLIVEIRA, V.R.; VIEIRA, C. \& TSAI, S.M. Nodulação em cultivares de feijão dos conjuntos gênicos andino e meso-americano. Pesq. Agropec. Bras., 37:1145-1150, 2002.

GILLER, K. E. \& WILSON, K. J. Nitrogen fixation in tropical cropping systems. Wallingford, CAB, 1993. 313p.

HARA, F.A.S. \& OLIVEIRA, L.A. Características fisiologicas e ecologicas de isolados de rizobios oriundos de solos ácidos de Iranduba, Amazonas. Pesq. Agropec. Bras., 40:667$672,2005$.
HOAGLAND, D.R. \& ARNON, D.I. The water culture method of growing plants without soil. Berkeley, University of California, 1950. 32p.

HUNGRIA, M.; VARGAS, M.A.T. \& ARAÚJO, R.S. Biologia dos solos dos cerrados. Planaltina, Embrapa-CNAC, 1997. $524 \mathrm{p}$.

HUNGRIA, M. \& BOHRER, T.R.J. Variability of nodulation and dinitrogen fixation capacity among soybean cultivars. Biol. Fert. Soils, 31:45-52, 2000.

HUNGRIA, M.; CAMPO, R.; CHUEIRE, L.; GRANGE, L. \& MEGÍAS, M. Symbiotic effectiveness of fast-growing rhizobial strains isolated from soybean nodules in Brazil. Biol. Fert. Soils, 35:387-394, 2001.

HUNGRIA, M.; CHUEIRE, L.M.O.; COCA, R.G.; MEGIAS, M. Preliminary characterization of fast growing rhizobial strains isolated from soybean nodules in Brazil. Soil Biol. Biochem., 33:1349-1361, 2001.

LIMA, A.S.; PEREIRA, J.P.A.R. \& MOREIRA, F.M.S. Diversidade fenotípica e eficiência simbiótica de estirpes de Bradyrhizobium spp. de solos da Amazônia. Pesq. Agropec. Bras., 40:1095-1104, 2005.

MOREIRA, F.M.S. \& SIQUEIRA, J.O. Fixação biológica do nitrogênio. In: MOREIRA, F.M.S. \&. SIQUEIRA, J.O. Microbiologia e bioquímica do solo. 2.ed. Lavras, Universidade Federal Lavras, 2006. p.501-529.

NEVES, M.C.P.; MARTINS, L.M.; XAVIER, G.R. \& RUMJANEK, N.G. Levantamento de estirpes de rizóbio capazes de nodular caupi (Vigna unguiculata) em solos do Nordeste do Brasil. I. Sertão. Seropédica, Embrapa Agrobiologia, 1998a. 10p. (Embrapa-CNPAB. Documentos, 46).

NEVES, M.C.P.; MARTINS, L.M.; XAVIER, G.R. \& RUMJANEK, N.G. Levantamento de estirpes de rizóbio capazes de nodular caupi (Vigna unguiculata) em solos do Nordeste do Brasil. II. Zona da Mata. Seropédica, Embrapa Agrobiologia, 1998b. 8p. ( Embrapa-CNPAB. Documentos, 47).

NOGUEIRA, C.O.N. Eficiência agronômica de Rizóbios selecionados e diversidade das populações nativas que Nodulam o feijoeiro-comum em Formiga- MG. Lavras, Universidade Federal de Lavras, 2005. (Tese de Mestrado)

SAEG. Sistema para análises estatísticas e genéticas, versão 8.0, Viçosa MG, Fundação Arthur Bernardes, 2000.

SANTOS, D.R. Seleção de estirpes de Bradyrhizobium sp. para fixação de dinitrogênio em caupi (Vigna unguiculata (L.) Walp.), em solos salinizados do semiárido. Recife, Universidade Federal Rural de Pernambuco, 1987. (Tese de Mestrado)

SANTOS, J.O. Divergência genética em feijão-fava (Phaseolus lunatus L.): Diversidade genética entre isolados nativos de rizóbios noduladores do feijão-fava (Phaseolus lunatus L.). Teresina, Universidade Federal do Piauí, 2008. (Tese de Mestrado) 
SILVEIRA, J.A.G.; CONTADO, J.L.; MAZZA, J.L.M. \& OLIVEIRA, J.T.A. Phosphoenolpyruvate carboxylase and glutamine synthetase activities in relation to nitrogen fixation in cowpea nodules. R. Bras. Fisiol. Veg., 10:9-23, 1998.

SOUZA, R.A.; HUNGRIA, M.; FRANCHINI, J.C.; MACIEL, C.D.; CAMPO, R.J. \& ZAIA, D.A.M. Conjunto mínimo de parâmetros para avaliação da microbiota do solo e da fixação biológica do nitrogênio pela soja. Pesq. Agropec. Bras., 43:83-91, 2008.

TRINICK, M.J. Relationships amongst the fast-growing rhizoia of Lablab purpureus, Leucaena leucocephala, Mimosa spp., Acacia farnesiana and Sesbania grandiflora and their afinities with other rhizobial groups. J. Appl. Bact., 49:39-53, 1980.

VINCENT, J.M. A manual for the practical study of roootnodule bacteria. Oxford, Blackwell Scientific, 1970. 164p. (International Biological Programme Handbook, 15)
XAVIER, G.R.; MARTINS, L.M.V.; RIBEIRO, J.R.A. \& RUMJANEK, N.G. Especificidade simbiótica entre rizóbios e acessos de feijão-caupi de diferentes nacionalidades. Caatinga, 19:25-33, 2006.

ZAMAN-ALLAH, M.; SIFI, B.; LTAIEF, B.; EL AOUNI, M.H. \& DREVON, J.J. Rhizobial inoculation and P fertilization response in common bean (Phaseolus vulgaris) under glasshouse and field conditions. Exper. Agric., 43:67-77, 2007.

ZILLI, J.É.; VALICHESKI, R.R.; RUMJANEK, N.G.; SIMÕESARAÚJO, J.L.; FREIRE FILHO, F.R.; NEVES, M.C.P. Eficiência simbiótica de estirpes de Bradyrhizobium isoladas de solo do Cerrado em caupi. Pesq. Agropec. Bras. 41: 811-818, 2006. 